\title{
Monocytes/macrophages activation contributes to b-gamma-glutamyltransferase accumulation inside atherosclerotic plaques
}

Eugenia Belcastro ${ }^{1,2}$, Maria Franzini ${ }^{1}$, Silvana Cianchetti ${ }^{3}$, Evelina Lorenzini', Silvia Masotti ${ }^{4}, V^{1}$ anna Fierabracci ${ }^{1}$, Angela Pucci ${ }^{5}$, Alfonso Pompella ${ }^{1}$ and Alessandro Corti ${ }^{1^{*}}$

\begin{abstract}
Background: Gamma-glutamyltransferase (GGT) is a well-established independent risk factor for cardiovascular mortality related to atherosclerotic disease. Four GGT fractions have been identified in plasma, but only b-GGT fraction accumulates in atherosclerotic plaques, and correlates with other histological markers of vulnerability. The present study was aimed to evaluate whether macrophagic lineage cells may provide a source of b-GGT within the atherosclerotic plaque.

Methods: GGT expression and release were studied in human monocytes isolated from peripheral blood of healthy donors. The growth factors GM-CSF and M-CSF were used to induce differentiation into M1-like and M2-like macrophages, respectively. Plaque GGT was investigated in tissue samples obtained from patients undergoing carotid endoarterectomy.

Results: We found that M1-like macrophages express higher levels of GGT as compared to M2-like, and that both monocytes and M1-like macrophages-but not M2-like-are able to release the b-GGT fraction upon activation with pro-inflammatory stimuli. Western blot analysis of b-GGT extracted from plaques confirmed the presence of a GGT immunoreactive peptide coincident with that of macrophages.

Conclusions: Our data indicate that macrophages characterized by a pro-inflammatory phenotype may contribute to intra-plaque accumulation of b-GGT, which in turn may play a role in the progression of atherosclerosis by modulating inflammatory processes and favouring plaque instability.
\end{abstract}

Keywords: Gamma-glutamyltransferase, b-GGT fraction, Monocytes, Macrophages, Atherosclerosis

\section{Background}

Monocyte/macrophage lineage cells represent one of the main cellular components of atherosclerotic lesions. Atherosclerosis is a progressive and chronic inflammatory disease characterized by cellular and molecular alterations within the arterial wall which lead to lumen narrowing and/or to plaque rupture with superimposed acute thrombosis $[1,2]$. Such condition is by far the most frequent underlying cause of coronary, carotid and

\footnotetext{
*Correspondence: alessandro.corti@med.unipi.it

1 Department of Translational Research and New Technologies in Medicine and Surgery, Medical School, University of Pisa, Via Roma 55, 56126 Pisa, Italy

Full list of author information is available at the end of the article
}

peripheral arterial disease [3]. The inflammatory reactions taking place in plaques implicate highly complex processes that are still not completely understood. However, it is known that the recruitment of leukocytes play a key role in these events, and monocytes in particular invade atherosclerotic lesions and differentiate into macrophages. The heterogeneity of macrophages detectable in atherosclerotic lesions has been a topic of great interest lately, with particular reference to the two major macrophage subpopulations involved in pro-inflammatory processes ("M1" phenotype) and in resolution and repair ("M2" phenotype), respectively. Actually, M1 and M2 macrophages are thought to represent the extreme polarization phenotypes of a continuum of pro- and 
anti-inflammatory macrophages all simultaneously present in atherosclerotic lesions [4-6]. M1 macrophages were demonstrated to be the predominant phenotype in rupture prone shoulder regions of human plaques, while M2 macrophages-associated markers were predominant in the adventitia [7]. On the other hand M2 macrophages were identified in more stable cell-rich areas, away from the lipid core of human atherosclerotic plaques $[8,9]$. It was suggested that-in response to the changing environment in developing atherosclerotic plaquesmacrophages may shift transiently between different phenotypes. Nevertheless a number of questions remain to be answered, e.g. little is known about the propensity of individual macrophage phenotypes to become foam cells, even if some reports suggest that both M1 and/or M2 macrophages may be foam cell precursors $[5,10]$.

Epidemiological studies showed that total serum gamma-glutamyltransferase (GGT) activity is an independent risk factor for cardiovascular mortality related to atherosclerotic disease [11] and a correlation between plasma GGT levels and the presence of multivessel disease has been proposed [12]. We recently identified four different GGT fractions in human blood [13] and an association between the fraction with highest molecular weight (b-GGT) with known cardiovascular risk factors-such as LDL-cholesterol, triglycerides, glucose and C-reactive protein-was demonstrated [14]. More recently, a first characterization revealed that b-GGT consists of membrane microvesicles with physical properties resembling those of exosomes [15].

Importantly, b-GGT has been detected also inside atherosclerotic plaques [16] and it has been suggested that this may be a factor in pathogenesis of atherosclerosis. Indeed, GGT enzyme activity is involved in the modulation of inflammatory mediators such as leukotrienes [17] and S-nitrosoglutathione (GSNO) [18] and it has been repeatedly documented that in the presence of glutathione and transition metal cations (in particular $\mathrm{Fe}^{3+}$ and $\mathrm{Cu}^{2+}$ ), GGT activity is able to trigger the production of reactive oxygen species (superoxide, hydrogen peroxide), thus promoting LDL oxidation and other pro-oxidant reactions potentially involved in the progression of atherosclerotic lesions [19-21]. The necrotic core of the plaque, where both GGT $[19,20]$ and iron [22] are present at adequate concentrations, represents an environment favourable to the evolution of such reactions.

Human mononuclear cells have long been known to express GGT activity [23, 24] and GGT-positive CD68+ macrophage-derived foam cells are present in the intimal layers of human atherosclerotic plaques $[25,26]$. The levels of intra-plaque b-GGT activity were demonstrated to correlate with both plasma b-GGT and levels of macrophagic infiltration, a histological marker of plaque vulnerability [27]. Against this background, the aim of the present study was to assess whether monocytes/macrophages may represent a source of b-GGT within the atherosclerotic plaque.

\section{Methods \\ Chemicals}

Unless otherwise indicated, all reagents were from Sigma Chemical Co. (St. Louis, MO, USA). Recombinant human cytokines and growth factors were purchased from PeproTech (London, UK).

\section{Ethics, consent and permissions}

The study was approved by the Institutional Ethics Committee of the University Hospital of Pisa, and conformed to the Declaration of Helsinki. Patients gave their verbal informed consent to participate and the data were analysed anonymously.

\section{Isolation and activation of monocytes}

Human peripheral blood mononuclear cells (PBMCs) were isolated by Histopaque ${ }^{\circledR}-1077$ density centrifugation from the blood of healthy donors. Monocytes were enriched by plating $\left(10 \times 10^{6}\right.$ cells/wells $)$ PBMCs in 6-well plates (Sarstedt) with RPMI 1640 medium containing $2 \mathrm{mmol} / \mathrm{L}$ L-glutamine and $10 \% \mathrm{v} / \mathrm{v}$ fetal calf serum, the latter ultracentrifuged $(100,000 \times g, 120 \mathrm{~min}$, $4{ }^{\circ} \mathrm{C}$ ) before addition to remove serum-associated b-GGT (data not shown). After $2 \mathrm{~h}$, non-adherent cells were removed and adherent cells were washed five times with RPMI 1640. Cytospin preparations of trypsinized cells were prepared to evaluate the purity of adherent monocytes $(74.5 \% \pm 3.4$ monocytes, $25.0 \% \pm 3.6$ lymphocytes, $1.1 \% \pm 0.4$ neutrophils; $\mathrm{n}=8$ ). Monocytes-enriched cell preparations were then incubated with ionomycin $(1 \mu \mathrm{M}$, $15 \mathrm{~min})$, LPS ( $5 \mu \mathrm{g} / \mathrm{ml}, 24 \mathrm{~h}$ ) or a combination of TNF $\alpha /$ IL-1 $\beta$ (both $10 \mathrm{ng} / \mathrm{ml}, 24 \mathrm{~h}$ ), as described by others [2830]. All cells were kept in a humidified incubator with $5 \%$ $\mathrm{CO}_{2} / 95 \%$ air and cell viability was assessed by Trypan blue exclusion. Finally media were collected and centrifuged at $300 \times g\left(5 \mathrm{~min}, 4^{\circ} \mathrm{C}\right)$ and $10,000 \times g\left(10 \mathrm{~min}, 4{ }^{\circ} \mathrm{C}\right)$ before GGT determinations. Further details are reported in the figure legends.

\section{Polarization of monocytes into M1-like or M2-like phenotype}

In vitro differentiation of monocytes into macrophages with properties similar to M1 and M2 cells ("M1-like" and "M2-like", respectively) was obtained as repeatedly described [10, 31-34]. Briefly, PBMCs isolated by Histopaque ${ }^{\circledR}-1077$ density centrifugation were left to adhere for $24 \mathrm{~h}$ in RPMI 1640 medium integrated with $2 \mathrm{mmol} / \mathrm{L} \mathrm{L}$-glutamine, $100 \mathrm{U} / \mathrm{ml}$ penicillin, $100 \mu \mathrm{g} / \mathrm{ml}$ 
streptomycin and $10 \% \mathrm{v} / \mathrm{v}$ fetal calf serum. After $24 \mathrm{~h}$, non-adherent cells were removed, while adherent cells were cultured for additional 6 days in complete medium integrated with $50 \mathrm{ng} / \mathrm{ml}$ recombinant human granulocyte/macrophage colony stimulating factor (GM-CSF) or with $50 \mathrm{ng} / \mathrm{ml}$ macrophage colony stimulating factor (M-CSF), respectively. Where indicated, cells were incubated for additional $24 \mathrm{~h}$ with a combination of TNF $\alpha /$ IL-1 $\beta$ (both $10 \mathrm{ng} / \mathrm{ml}$ ) [28-30] and media were collected as described above.

In another set of experiments, adherent monocytes were incubated with M-CSF for 6 days in the presence of a mouse anti-IL10 antibody $(1 \mu \mathrm{g} / \mathrm{ml}$; Abcam) or the corresponding isotype control ( $1 \mu \mathrm{g} / \mathrm{ml}$; Abcam). Other experiments were finally performed by incubating adherent monocytes with TNF $\alpha(10 \mathrm{ng} / \mathrm{ml})$ and/or $\mathrm{IL}-10$ $(20 \mathrm{ng} / \mathrm{ml})$ in complete medium for $48 \mathrm{~h}$. Where indicated, cells were pre-treated $(20 \mathrm{~min})$ with IL-10, then $\mathrm{TNF} \alpha$ was added to incubation media. Cell viability was assessed by Trypan blue exclusion. Further details are reported in the figure legends.

\section{Plaques and monocytes staining}

Samples of carotid plaques were from patients undergoing carotid endarterectomy at the General and Vascular Surgery (University Hospital, Pisa, Italy) and were already fully characterized [27]. The patients were asymptomatic, since endarterectomy is recommended in case of severe carotid artery stenosis $(>70 \%)$ even in absence of neurological symptoms [35]. Surgically excised carotid plaques were collected on ice and dissected into 5 - $\mathrm{mm}$ segments, then a segment of the plaque was frozen at $-20{ }^{\circ} \mathrm{C}$ in Optimal Cutting Temperature (OCT) medium for in situ evaluation of enzymatic activity. Plaque sections were stained as described [27]. Briefly, serial $3 \mu \mathrm{m}$ paraffin sections were stained with hematoxylin-eosin and Masson's trichrome method. Immunohistochemistry was performed on adjacent paraffin-embedded sections. Macrophages were identified by immunoperoxidase staining by using a specific monoclonal antibody raised against CD68 antigen (Dakopatts, Glostrup, Denmark) and the immunoreaction was visualized by 3-diaminobenzidine substrate. GGT immunostaining was performed by using a previously characterized polyclonal antibody directed against the heavy chain of human GGT antibody at the appropriate dilution (1:1600) [36]. Basing on plaque histology [27], two $(\# 1, \# 2)$ representative thin-cap fibroatheromas with large necrotic core and medium-high macrophage infiltration score, and one (\#3) stable, thickcap fibroatheroma with small necrotic scores and low macrophage infiltration score, were selected (Table 1).

Cytochemical staining for GGT activity was performed on air-dried buffy coat films fixed in a phosphatebuffered acetone formaldehyde mixture (PBAF) and incubated with GGT substrate gamma-glutamyl-4-methoxy-2-naphtylamide and Fast Garnet GBC, as previously described [24]. Nuclei were counterstained with methyl green.

\section{Microvesicles isolation}

Portions of plaque tissue were snap-frozen in liquid nitrogen, mechanically disrupted and homogenized in $5 \%$ volume of cold hypotonic buffer (PBS diluted 10 folds) using a Potter homogenizer. Isolation of microparticles and exosomes from monocytes/macrophages supernatants as well as from homogenates of atherosclerotic lesions was performed by a differential centrifugation procedure [15]. Samples were first centrifuged at $2000 \times g$ for $10 \mathrm{~min}$ at $4{ }^{\circ} \mathrm{C}$, then supernatants were centrifuged at $10,000 \times g\left(45 \mathrm{~min}, 4{ }^{\circ} \mathrm{C}\right)$ to remove large debris. Again, supernatants were collected, transferred into ultracentrifuge tubes and centrifuged at $100,000 \times g\left(120 \mathrm{~min}, 4^{\circ} \mathrm{C}\right)$. Pellets were then washed $\left(100,000 \times g, 120 \mathrm{~min}, 4{ }^{\circ} \mathrm{C}\right)$ and finally resuspended in physiological saline.

\section{Western blot analysis}

For western blot determinations of GGT, isolated monocytes, lymphocytes, platelets and cultured endothelial HMEC-1 cells-harvested in hypotonic lysis buffer (10 mM Tris-HCl, pH 7.8)—or plaque derived exosomes were used. Non-adherent lymphocytes were isolated from plated PBMCs, whereas platelets were obtained from buffy-coat derived platelet concentrates. All samples were thoroughly washed in order to remove

Table 1 Histological features of the selected plaques used

\begin{tabular}{|c|c|c|c|c|c|c|c|}
\hline \multirow[t]{2}{*}{ N. } & \multirow[t]{2}{*}{ Cap thickness } & \multirow[t]{2}{*}{ Necrotic core } & \multirow[t]{2}{*}{ CD68+ macrophages } & \multirow[t]{2}{*}{ Fibrous tissue } & \multirow[t]{2}{*}{ Calcification } & \multicolumn{2}{|c|}{$\begin{array}{l}\text { Intra-plaque activities } \\
\text { (mU/g tissue) }\end{array}$} \\
\hline & & & & & & b-GGT & Total GGT \\
\hline$\# 1$ & Thin and ulcerated & High & Medium & Low & Low & 28.89 & 100.73 \\
\hline \#2 & Thin and ulcerated & High & Medium & Low & Low & 14.61 & 47.02 \\
\hline \#3 & Thick & Medium & Low & Medium & High & 9.43 & 35.58 \\
\hline
\end{tabular}


contaminating plasma GGT. Endothelial HMEC-1 cells were grown in MCDB 131 medium (Life Technologies) supplemented with $1 \mu \mathrm{g} / \mathrm{ml}$ hydrocortisone, $10 \mathrm{ng} / \mathrm{ml}$ EGF (Life Technologies) and $10 \% \mathrm{v} / \mathrm{v}$ fetal calf serum, and cultured at $37{ }^{\circ} \mathrm{C}$ in a $5 \% / 95 \% \mathrm{CO}_{2} /$ air atmosphere.

All samples were separated by $8 \%$ SDS-PAGE [37] and incubated with rabbit anti-GGT IgG directed against the C-terminal 20 amino acids of human GGT heavy chain prepared as described [36]. Visualization of protein bands was obtained using a horseradish peroxidase-conjugated anti-rabbit IgG antibody (Santa Cruz Biotechnology, Santa Cruz, CA, USA) and the ECL detection system (Roche, Basel, Switzerland). Bands were analyzed with a Bio-Rad ChemiDoc apparatus equipped with the QuantityOne software.

\section{Fractional GGT analysis by high-performance gel filtration chromatography}

Determination of GGT fractions was performed as previously described $[13,38]$ by a FPLC system (AKTA-purified-10, GE-Healthcare). Separation and quantification of GGT fractions was performed by gel-filtration chromatography (Superose 6 10/300, GE Healthcare) followed by post-column injection of the fluorescent substrate gamma-glutamyl-7-amido-4-methylcoumarin. Intensity of the fluorescence signal was expressed in arbitrary fluorescence units (f.u.) and the area under chromatographic peaks was proportional to GGT activity. The elution volume for b-GGT fraction is $12.9 \mathrm{ml}$, corresponding to a molecular weight (MW) of $2000 \mathrm{kDa}$ [13].

\section{Other determinations}

GGT activity was determined according to Huseby and Strömme [39]. Cytokines were measured using specific enzyme-linked immunosorbent assay kits (TNF- $\alpha, R \& D$ Systems; IL-6 and IL-10, Affymetrix eBioscience) according to the manufacturer's instructions. Protein content was determined by the Bradford's method using the BioRad protein assay reagent. Statistical analysis of data was performed by Student's $t$ test for paired observations and one-way ANOVA with Newman-Keuls test for multiple comparisons.

\section{Results}

\section{Monocytes activation}

In agreement with earlier evidence [24], different levels of GGT activity were detected when monocytes isolated from the blood of healthy donors were stained for the enzyme (Fig. 1a), and a mean value of $10.1 \pm 2.0 \mathrm{mU} /$ $\mathrm{mg}$ of protein in the whole homogenate was calculated. In order to understand whether such cells were able to release GGT, isolated monocytes were exposed to activating substances and GGT activity was measured in the incubation media. As reported in Fig. 1, both the combination of pro-inflammatory cytokines TNF $\alpha / \mathrm{IL}-1 \beta$ (Fig. 1b) and bacterial lipopolysaccharide (LPS; Fig. 1c) significantly increased $(\mathrm{p}<0.05)$ GGT activity in the incubation media as compared to controls. In a separate set of experiments also the calcium ionophore ionomycin induced a significant $(\mathrm{p}<0.05)$ release of GGT activity in the incubation media (data not shown). GGT activity was also detected in the media of the corresponding control samples (Fig. 1b, c), possibly ensuing from a weak activation of monocytes during isolation/incubation procedures. As regard cell-associated GGT activity, TNF $\alpha /$ IL-1 $\beta$ treatment did not produce any appreciable effect (Fig. 1d), whereas a significant decrease was induced by LPS (Fig. 1e; p < 0.05).

\section{Macrophages activation}

M1- and M2-like macrophages were obtained by incubating aliquots of monocytes (isolated from the same buffy coat) with GM-CSF or M-CSF, respectively. As previously described [32], after 6 days of culture the majority of M1-like macrophages presented with a classical adherent, "fried egg" morphology, whereas M2-like macrophages primarily presented with a stretched, spindle-like morphology (data not shown). The analysis of cytokines concentration in culture media revealed significantly $(\mathrm{p}<0.01)$ higher levels of TNF $\alpha$ and IL-6 (Fig. 2a, b) and lower ( $<$ < 0.05) levels of IL-10 (Fig. 2c) for M1-like as compared to M2-like macrophages. M1-like macrophages showed also significantly $(\mathrm{p}<0.005)$ higher levels of cell associated GGT activity (Fig. 3a) and protein (Fig. 3b) as compared to M2-like macrophages. SDS-PAGE analysis revealed that the GGT heavy subunit presented with the same apparent MW in both differentiated macrophages and monocytes (Fig. 3b).

The exposure to pro-inflammatory cytokines TNF $\alpha /$ IL-1 $\beta$ induced a significant increase $(\mathrm{p}<0.05)$ of both released (Fig. 4a) and cell-associated GGT (Fig. 4c) only in M1-like macrophages, whereas no appreciable effect was detectable in M2-like cells (Fig. 4b, d).

\section{Regulation of GGT expression in M1-like and M2-like macrophages}

In order to characterize the possible mechanisms underlying the lower expression of GGT in M2-like macrophages, we evaluated the involvement of IL-10. As expected, $48 \mathrm{~h}$ incubation of monocytes with TNF $\alpha$ resulted in increased levels of cellular GGT activity (Fig. 5a) and protein (data not shown), but this effect was completely abolished when also IL-10 was added to incubation media (Fig. 5a). In a separate set of experiments, M2-like macrophages were incubated during their 

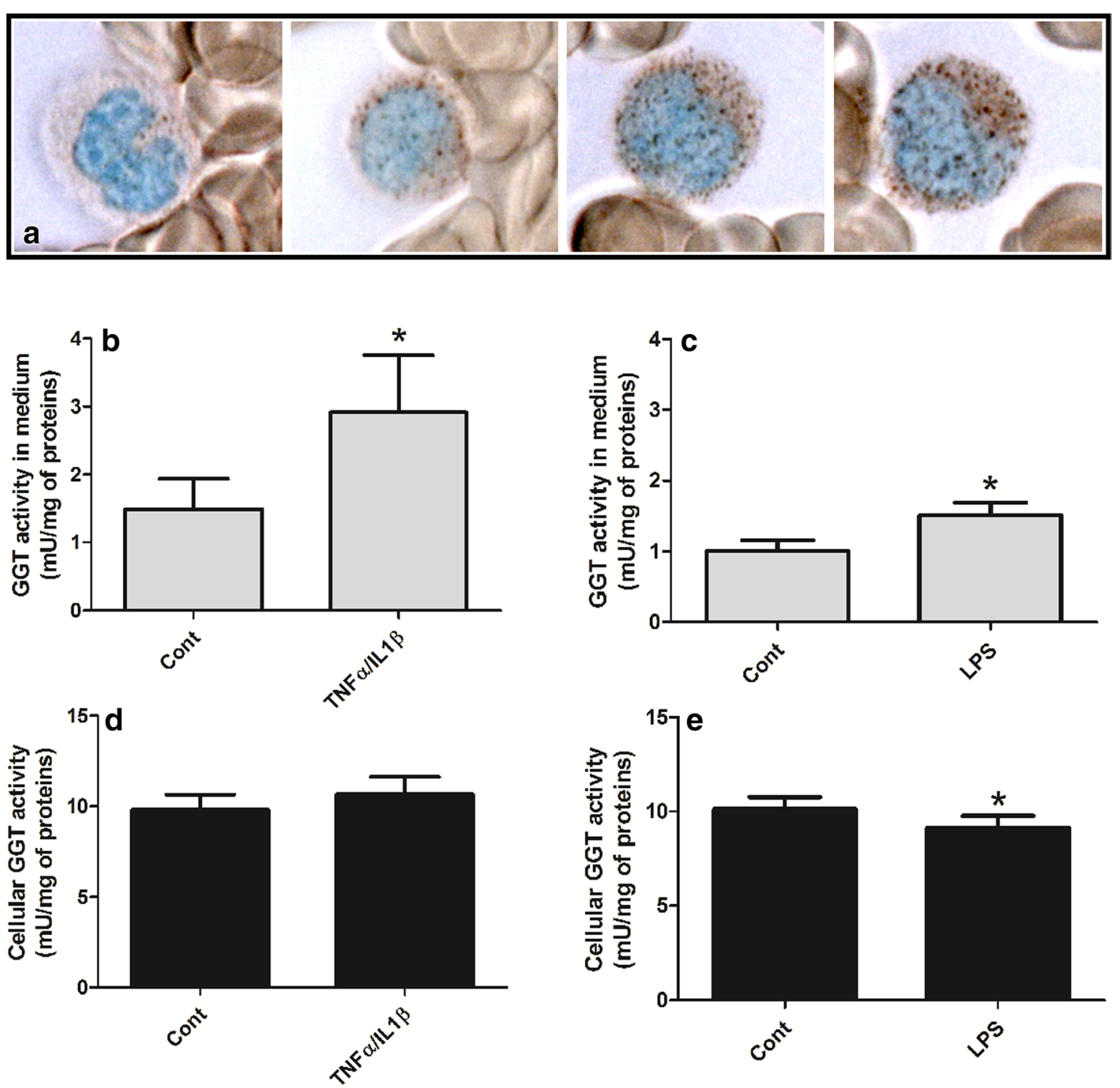

Fig. 1 Cytochemical staining for GGT enzyme activity expressed in activated monocytes. Monocytes from the blood of healthy donors expressing different levels of GGT activity. The azo-dye reaction product (reddish-brown) varies in intensity from slight to very strong, resulting in diffuse staining or granular positivity. Nuclei were counterstained with methyl green. Original magnification: $\times 100$ (a). Monocytes isolated from fresh buffy coats were incubated in the presence of TNF $\alpha / \mathrm{LL}-1 \beta$ (both $10 \mathrm{ng} / \mathrm{ml} ; 24 \mathrm{~h}$ ) or LPS $(5 \mu \mathrm{g} / \mathrm{ml} ; 24 \mathrm{~h}$ ). GGT activity was measured in the $10,000 \times g$ centrifuged supernatants $(\mathbf{b}, \mathbf{c})$ and in cellular homogenates $(\mathbf{d}, \mathbf{e})$. Results — expressed as $\mathrm{mU} / \mathrm{mg}$ of cellular proteins - are mean \pm SD of five to nine separate determinations. Data were analyzed by Student's $t$ test for paired data; ${ }^{*} p<0.05$ as compared with the corresponding control

differentiation with an anti-IL-10 antibody. As shown in Fig. 5b the addition of the blocking antibody was associated with induction of significantly higher levels of both GGT activity $(\mathrm{p}<0.05)$ and protein (data not shown).

\section{Release of b-GGT fraction in monocytes/macrophages supernatants}

Incubation media from activated monocytes and M1-like macrophages were ultracentrifuged at $100,000 \times g$ and incubation media were analyzed by gel-filtration chromatography. As shown in Fig. 6 a peak corresponding to the b-GGT fraction was detected in the incubation media of both monocytes (Fig. 6a) and M1-like macrophages (Fig. 6b). In both cases, the exposure to the pro-inflammatory cytokines TNF $\alpha / \mathrm{IL}-1 \beta$ increased the release of b-GGT.

\section{GGT expression in atherosclerotic plaque}

In agreement with previous evidence [26, 27], the immunohistochemical staining of plaques sections revealed the 


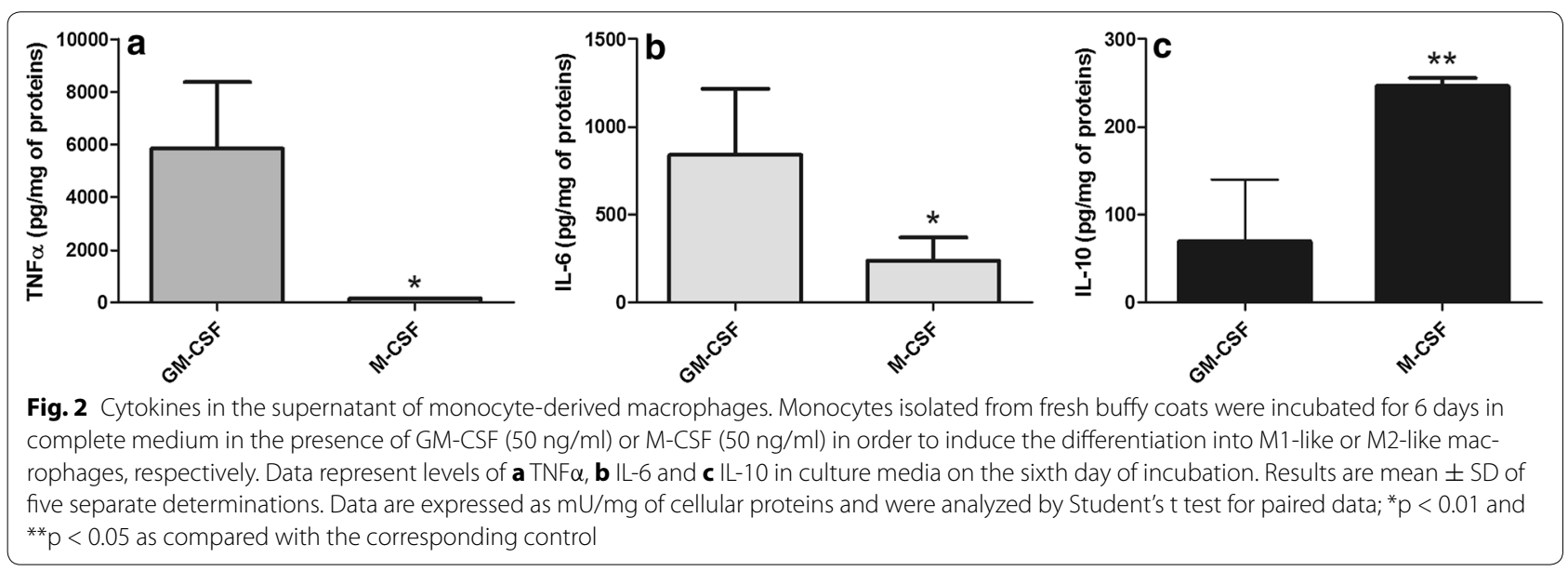

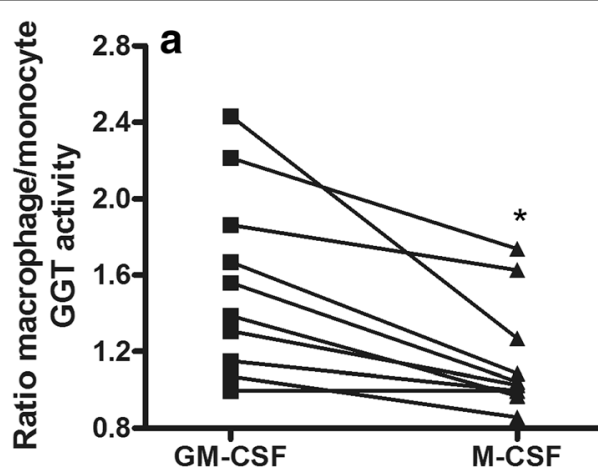

b Monocytes GM-CSF M-CSF

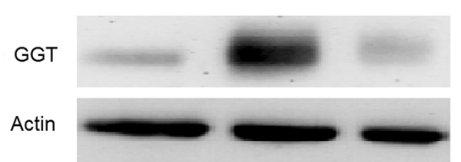

Fig. 3 Effects of GM-CSF and M-CSF exposure on cellular GGT expression. Monocytes isolated from fresh buffy coats were incubated for 6 days in complete medium in the presence of GM-CSF $(50 \mathrm{ng} / \mathrm{ml})$ or M-CSF $(50 \mathrm{ng} / \mathrm{ml})$ in order to induce the differentiation into M1-like or M2-like macrophages, respectively. a Data are ratioes between GGT activities determined after differentiation and activities present in initial monocytes. The results of ten separate paired incubations are shown. Solid lines connect data pairs obtained with the same starting monocytes preparation. Statistical analysis was performed by Student's t test for paired data; ${ }^{*} p=0.0016$. b Representative western blot analysis of GGT expression. Lane 1 monocytes; lane 2 M1-like macrophages; lane $3 \mathrm{M} 2$-like macrophages

presence of variable amounts of immunoreactive GGT, mainly localized to areas corresponding to necrotic core and macrophage-rich sites (Fig. 7).

The fractional GGT analysis of plaque homogenates (Table 1) confirmed the presence of a major peak corresponding to the b-GGT fraction (Fig. 8a-c). In order to further characterize the properties of such fraction, plaque b-GGT was isolated by ultracentrifugation from all the selected samples, and the GGT protein was compared by SDS-PAGE with that expressed by monocytes, lymphocytes, platelets and endothelial cell line HMEC-1 (Fig. 8d). Using a specific antibody against the heavy subunit of GGT, a single GGT band with the same apparent molecular weight was observed in monocytes, lymphocytes and platelets, while two bands were present in HMEC-1 cells, a heavier one and one slightly lighter than those observed in the other cell types. Plaques homogenates showed three bands: one comparable with the heavier band of HMEC-1, one with the same apparent MW of monocytes, lymphocytes and platelets, and one lighter than all the other observed bands. Interestingly, the second band was clearly detectable only in samples with a higher macrophage score (\#1 and \#2).

\section{Discussion}

GGT activity is a well-established independent risk factor for coronary and cerebrovascular events both in unselected populations [11] and in patients with diagnosed coronary artery disease [12]. GGT activity has been detected inside human atherosclerotic plaques associated both to CD68+ macrophage-derived foam cells $[25,26]$ and to a microvesicles-like fraction called b-GGT $[16,27]$. The b-GGT fraction is the heaviest of the four GGT fractions identified in plasma [38] and it is the sole identified within plaque homogenates [27]. In a recent study b-GGT has been demonstrated to consist of membrane microvesicles [15], sharing the physical properties of exosomes. Interestingly, intra-plaque b-GGT activity correlates with the histological markers 

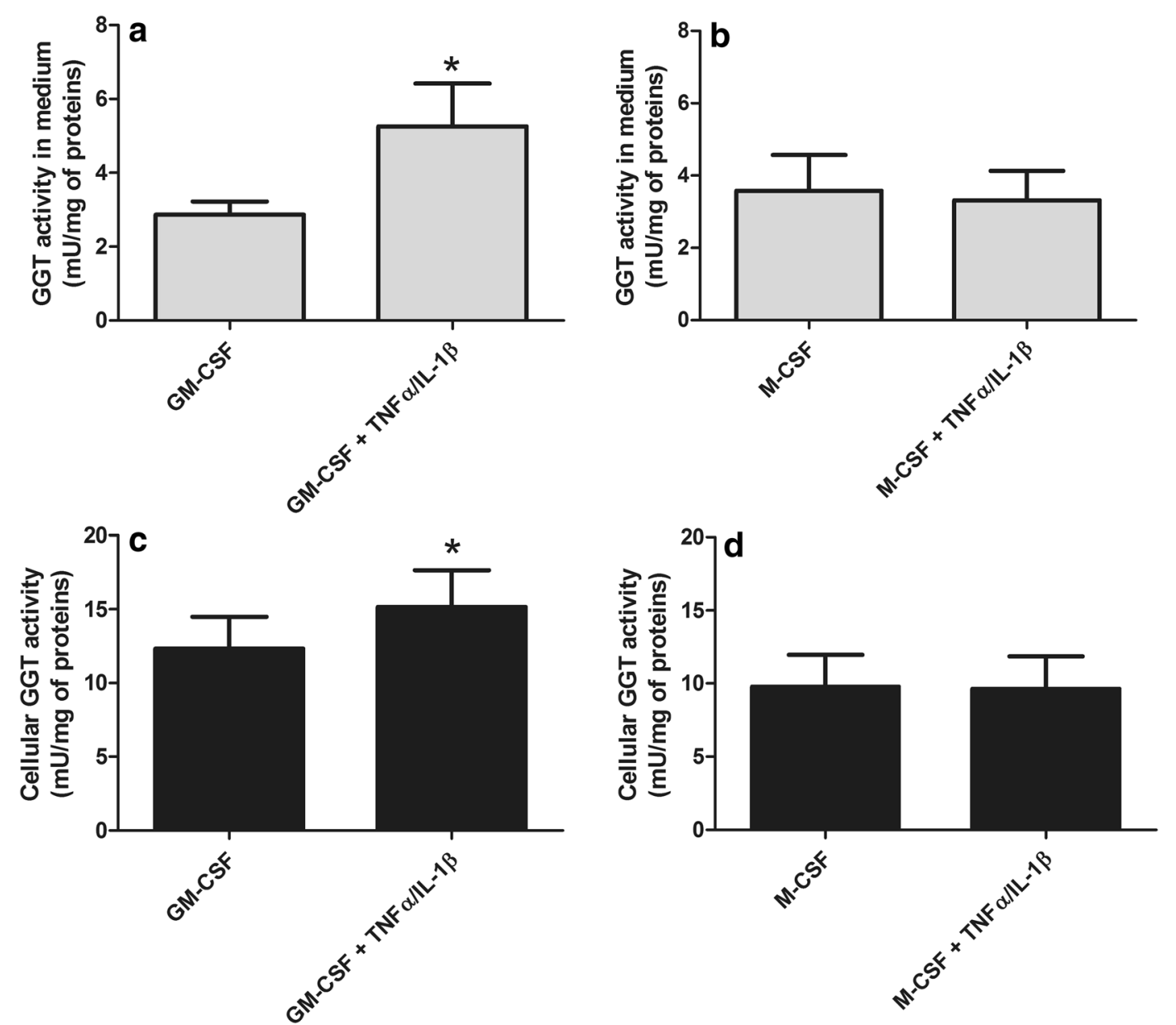

Fig. 4 GGT release by activated macrophages. Monocytes isolated from fresh buffy coats were incubated for 6 days in complete medium in the presence of GM-CSF $(50 \mathrm{ng} / \mathrm{ml})$ or M-CSF $(50 \mathrm{ng} / \mathrm{ml})$ in order to induce the differentiation into M1-like or M2-like macrophages, then they were incubated in the presence of TNF $\alpha / \mathrm{IL}-1 \beta$ (both $10 \mathrm{ng} / \mathrm{ml}$ ) for additional $24 \mathrm{~h}$. GGT activity was measured in the 10,000 $\times g$ centrifuged supernatants $(\mathbf{a}, \mathbf{b})$ and in cellular homogenates $(\mathbf{c}, \mathbf{d})$. Results are mean \pm SD of eight separate determinations. Data are expressed as mU/mg of cellular proteins and were analyzed by Student's $t$ test for paired data; ${ }^{*} p<0.05$ as compared with the corresponding untreated control

of plaque vulnerability-such as high macrophagic infiltration-as well as with levels of plasma b-GGT [17-27]. These pieces of evidence have prompted the need of identifying the origin of GGT accumulating inside atherosclerotic plaques, in order to better understand its role in plaque instability.

It was proposed that part of b-GGT present in atherosclerotic plaques might derive from the bloodstream through damaged endothelium [16, 27]. On the other hand an endogenous release of b-GGT by cellular elements of plaque-such as macrophages-is also conceivable. Indeed our results demonstrate that both monocytes (Figs. 1, 6) and pro-inflammatory M1-like macrophages (Figs. 4, 6) are able to release b-GGT upon activation. These results are in agreement with what already observed for neutrophils-whose contribute to inflammatory exudate b-GGT in cystic fibrosis airways has been assessed [37]- and adds evidence in support of the connection between GGT and inflammation. GGT is expressed by inflammatory cells [24] and its expression is induced by inflammatory cytokines (e.g. TNF $\alpha$, IFNs) and oxidative stress. Two major inflammatory mediators, cysteinyl-leukotriene LTC4 and S-nitrosoglutathione, are GGT substrates, and it was even proposed that soluble GGT may effect a cytokine-like function [40].

The potential contribution of macrophages to plaque b-GGT accumulation was further investigated by SDSPAGE analysis of samples belonging to a previously characterized series of carotid plaques [27]. A single 


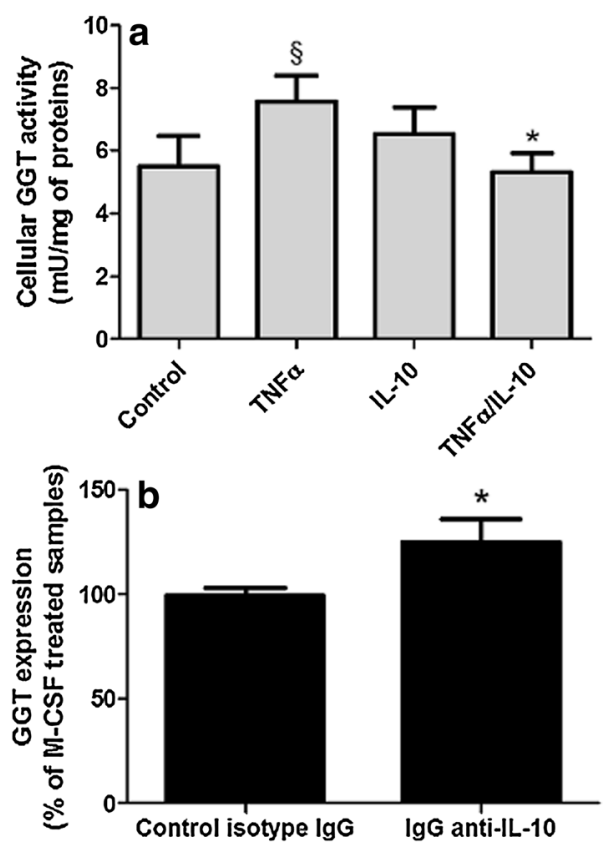

Fig. 5 Effects of TNF $\alpha$ and IL-10 on GGT expression in monocytes. a Freshly isolated monocytes were incubated with TNF $\alpha(10 \mathrm{ng} / \mathrm{ml})$ and/or IL-10 $(20 \mathrm{ng} / \mathrm{ml})$ in complete medium for $48 \mathrm{~h}$. Results are mean \pm SD of eight separate determinations. Data are expressed as $\mathrm{mU} / \mathrm{mg}$ of cellular proteins and were analyzed by one-way ANOVA with Newman-Keuls test for multiple comparisons; ${ }^{\S} \mathrm{p}<0.001$ as compared to untreated control; ${ }^{*} p<0.001$ as compared with TNF $\alpha$ treated samples. $\mathbf{b}$ Isolated monocytes were let to adhere for $24 \mathrm{~h}$, then they were incubated with M-CSF $(50 \mathrm{ng} / \mathrm{ml})$ in the presence of an anti-IL 10 antibody $(1 \mu \mathrm{g} / \mathrm{ml})$ or the corresponding isotype control for 6 days. Results are expressed as the ratio of GGT expressed by antibody-treated cells against M-CSF treatment alone (mean \pm SD of seven separate determinations). Data were analyzed by Student's $t$ test for paired data; ${ }^{*} p<0.05$

peak corresponding to b-GGT was detected in all the plaques studied (Fig. 8a-c), but SDS-PAGE analysis revealed a heterogeneous composition of such fraction (Fig. 8d). In this respect it should be considered that GGT protein is transcribed from a single gene (ggt1), nevertheless several distinct glycosylation forms have been described, resulting in different molecular weights [41]. It was also demonstrated that inflammatory cells present in the vessel wall might induce modifications in protein sialylation status [42]. The presence of different bands corresponding to distinct MWs (Fig. 8d) may thus reflect both a heterogeneous origin for intraplaque b-GGT as well as an intra-plaque modification of GGT protein (e.g. proteolysis, sialylation). SDSPAGE analysis nevertheless confirmed that inflammatory as well as endothelial cells might contribute to b-GGT accumulation within the atherosclerotic plaque.
SDS-PAGE does not allow discriminating among different "inflammatory" sources for b-GGT, and it is conceivable that macrophages, lymphocytes and platelets all can contribute to intra-plaque b-GGT. In particular, the demonstrated correlation between intra-plaque b-GGT activity and the levels of macrophages infiltration [27] and the fact that both monocytes (Figs. 1, 6) and M1-like macrophages (Figs. 4, 6) are able to release b-GGT upon activation, suggest that macrophages play a major role in b-GGT accumulation inside the atherosclerotic plaque.

As previously observed for neutrophilic infiltrates in cystic fibrosis [37], b-GGT accumulation in the atherosclerotic plaque should not be interpreted as the mere epiphenomenon of an exogenous contribute (i.e., from the bloodstream), or as the effect of inflammationrelated oxidative stress. Rather, the expression and the release of b-GGT by macrophages may be considered as one of the effects associated with the immune response, possibly involved in the modulation of selected mediators (e.g. GSNO, leukotrienes). Moreover, in selected conditions GGT activity has been shown to promote the production of reactive oxygen species and other prooxidants, resulting in redox reactions potentially involved in the progression of atherosclerosis [19-21]. M1 macrophage content of atherosclerotic plaques is positively associated with increased inflammation and clinical incidence of ischemic stroke [9]. High numbers of M1 macrophages were detected in symptomatic plaques, whereas smaller numbers, predominantly staining for M2 macrophage markers, were detected in asymptomatic plaques [5, 7-9]. The higher levels of GGT expression and b-GGT release observed from M1-like macrophages may thus play a role in plaque instability.

Altogether, our data indicate that inflammatory conditions are able to increase the expression and release of GGT enzyme, which in turn could play a role in modulating inflammation in the plaque environment. The higher cellular GGT expression in GM-CSFinduced M1-like macrophages, as compared to M-CSFinduced M2-like ones (Figs. 1a, b), appears to further support such hypothesis. Our data are in agreement with earlier evidence obtained with the human leukemic cell line KG-1, where incubation with GM-CSF resulted in a significant increase in GGT enzyme activity, while M-CSF did not produce any appreciable effect [43]. As far as treatment of monocytes with GM-CSF and M-CSF it has been reported that both GM-CSF and M-CSF receptors activate Ras-dependent signal transduction pathways and are both capable of activating AP-1 [44]. GM-CSF [45] and to some extent M-CSF 

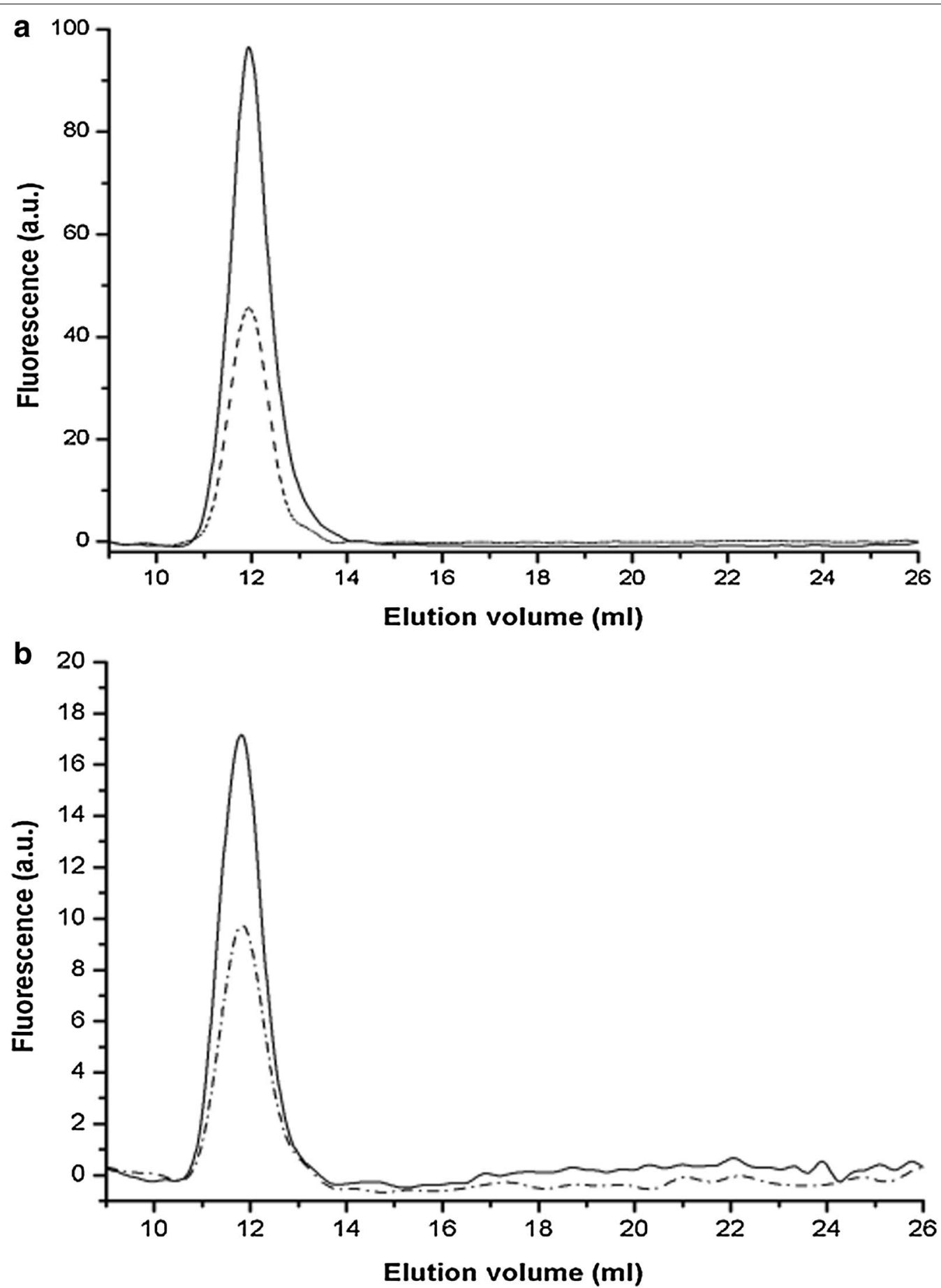

Fig. 6 Gel filtration chromatography of b-GGT released by TNF $\alpha /$ /L-1-activated monocytes (a) and M1-like macrophages (b). Monocytes and GMCSF-differentiated M1-like macrophages were incubated with TNF $\alpha / \mathrm{LL}-1 \beta$ (both $10 \mathrm{ng} / \mathrm{ml}, 24 \mathrm{~h}$ ), then supernatans were pooled and ultracentrifuged $\left(100,000 \times g, 120 \mathrm{~min}, 4^{\circ} \mathrm{C}\right)$. The resuspended final pellets were analyzed by gel filtration chromatography. Representative elution profiles of controls (dashed lines) and TNF $\alpha / \mathrm{IL}-1 \beta$ - activated cells (continuous line) are shown 

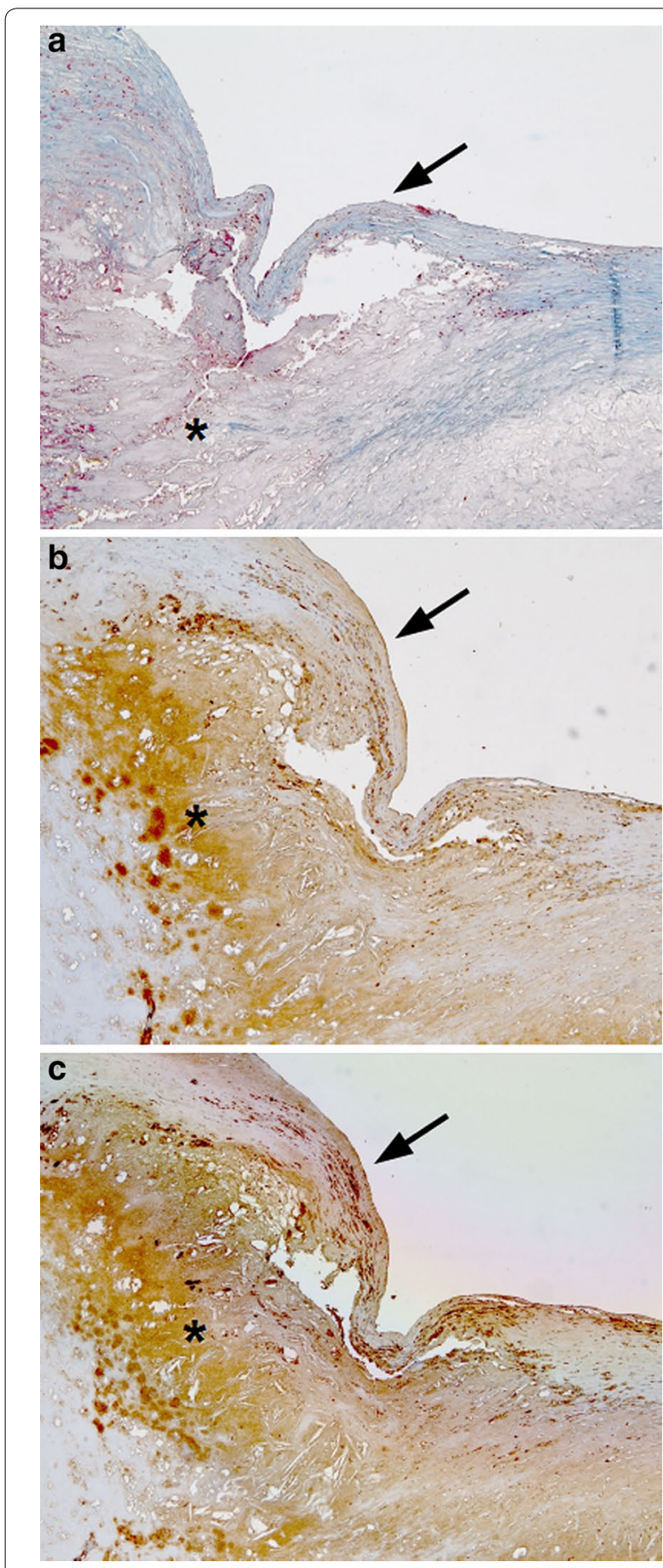

Fig. 7 GGT expression in atherosclerotic plaques. A representative thin cap fibroatheroma without ulceration is shown. Masson's trichrome staining (a) blue collagen fibers; red cytoplasm; black nuclei); immunohistochemistry for GGT protein (b) and macrophage-associated antigen CD68 (c). GGT and CD68 were localized by immunoperoxidase reaction with 3-diaminobenzidine as substrate, yielding a reddish-brown color. Immunoreactivities are mainly localized in the necrotic core (asterisk) extending just beneath the endothelial lining of the plaque (arrow). Sections were couterstained with haematoxylin. Original magnification: 10x
[46] are also known to modulate NF- $\mathrm{kB}$ activation, but with some important differences in terms of timing, duration and factors involved [47]. In agreement with previous evidence [33,47], our M1-like macrophages produced higher levels of pro-inflammatory TNF $\alpha$ and IL- 6 and lower levels of anti-inflammatory IL-10, whereas the opposite was observed with M2-like macrophages (Fig. 2). Indeed, it has been proposed that Ras signalling is involved in GGT expression [48], and synthesis of GGT mRNA is induced by cytokines-including TNF $\alpha$-through a NF-кB-dependent pathway [49, 50]. The differential basal expression of cytokines in M1-like and M2-like macrophages could thus account for the differential GGT expression observed. Indeed TNF $\alpha$ was able to significantly increase GGT expression in monocytes, but this effect was inhibited in the presence of IL-10 (Fig. 5a), i.e. a cytokine known to inhibit NF- $\kappa B$ pathway [51, 52]. Accordingly, when monocytes were differentiated into M2-like cells, the addition of an anti-IL-10 antibody was associated to higher levels of GGT expression (Fig. 5b). The lack of GGT induction in M2-like cells following TNF $\alpha / \mathrm{IL}-1 \beta$ might be therefore explained by such inhibitory effect of endogenous IL-10 (Fig. 4c, d), whose expression is markedly higher in M2- as compared to M1-like cells [47]. These observations support the hypothesis that the balance of pro-inflammatory/anti-inflammatory cytokines such as TNF $\alpha$ and IL-10 may differentially modulate GGT expression.

Besides GM-CSF or M-CSF treatments, macrophage M1 or M2 phenotypes have also been obtained by other in vitro procedures. M-CSF was shown to induce M1 differentiation in the presence of IFN- $\gamma$ and/or bacterial products such as LPS [53], whereas three distinct M2 subtypes were induced by treatments with IL-4 or IL-13 ("M2a" phenotype), or immune complexes in combination with IL-1 $\beta$ or LPS ("M2b"), or IL-10, TGF $\beta$ and glucocorticoids ("M2c") [54]. Interestingly, the ggt5 gene (formerly designated as GGTLA1/GGT-rel or GGL) was also recently shown to be highly expressed in GM-CSF differentiated macrophages [33]. GGT5 is a member of GGT family supposed to be mainly responsible for the conversion of LTC4 to LTD4 [55].

\section{Conclusions}

The results of the present study indicate that macrophages characterized by a pro-inflammatory phenotype can release b-GGT and contribute thus to its accumulation inside atherosclerotic plaques. Our observations may explain the existing association between b-GGT activity in thin-cap fibroatheromas and greater macrophage infiltration [27]. Further studies are however 

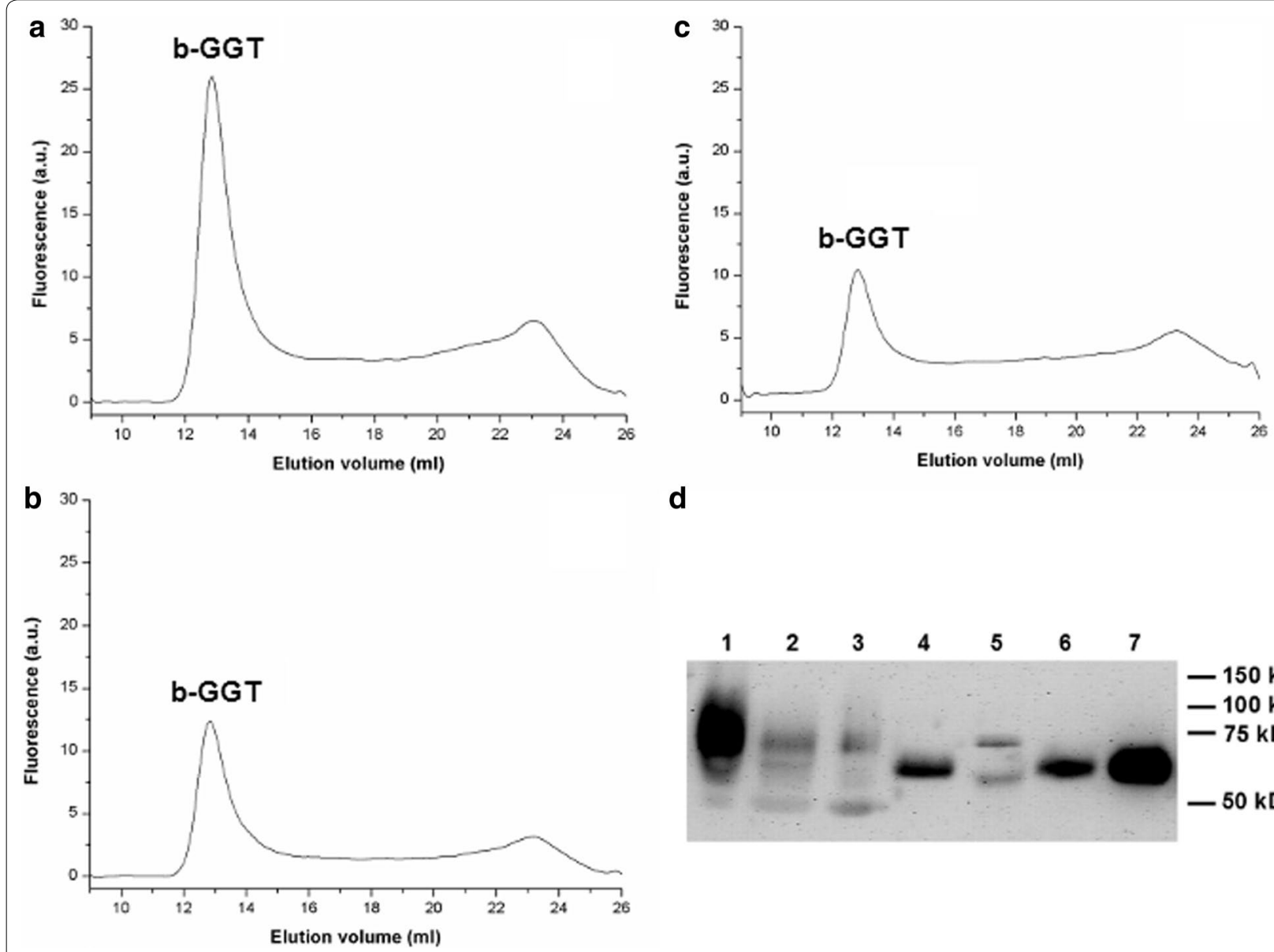

d

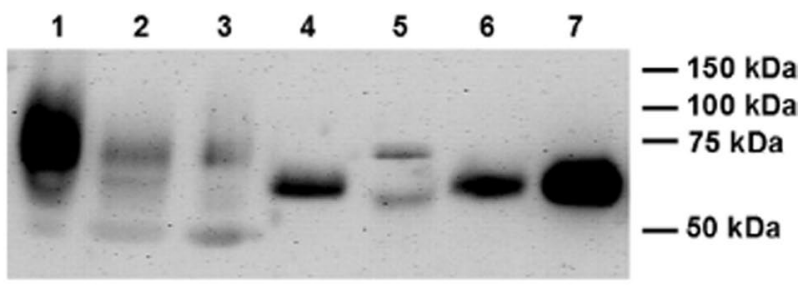

Fig. 8 Elution profile of GGT activity from whole homogenate of three selected plaque samples $(\mathbf{a}, \mathbf{b}, \mathbf{c})$. Elution profile was obtained by molecular exclusion chromatography, and GGT activity was detected by an online post-column reaction in the presence of a fluorescent substrate. A major peak of activity eluting at $12.9 \mathrm{ml}$ and corresponding to the b-GGT fraction is detectable. $\mathbf{d}$ Western blot analysis of GGT heavy chain in samples of isolated plaque b-GGT and other biological samples. Lanes 1-3 b-GGT from plaques \#1-3, respectively; lane 4 monocytes; lane 5 HMEC-1 endothelial cells; lane 6 lymphocytes; lane 7 platelets

needed to fully identify the different sources of intraplaque b-GGT and their actual role in the pathogenesis/ progression of atherosclerosis.

\section{Abbreviations}

AP-1: activator protein 1; b-GGT: big-gamma-glutamyltransferase; FPLC: fast protein liquid chromatography; GGT: gamma-glutamyltransferase; GM-CSF: granulocyte/macrophage colony stimulating factor; HMEC-1: human dermal microvascular endothelial cells; IL: interleukin; LPS: bacterial lipopolysaccharide; M-CSF: macrophage colony stimulating factor; NF-кB: nuclear factor kappa-light-chain-enhancer of activated B cells; PBMCs: peripheral blood mononuclear cells; TNF $\alpha$ : tumor necrosis factor alpha.

\section{Authors' contributions}

EB designed and performed the experiments, wrote and reviewed the manuscript; MF analyzed the data, wrote and reviewed the manuscript; SC conceived and designed the experiments; EL performed the experiments and analyzed data; SM performed chromatographic determinations and data analysis; VF analyzed the data; APu collected plaque samples and performed histopathological characterization; APo contributed to analysis and interpretation of data, drafted and reviewed the manuscript; AC conceived and designed the experiments, analyzed the data and drafted and reviewed the manuscript. All authors read and approved the final manuscript.

\section{Author details}

${ }^{1}$ Department of Translational Research and New Technologies in Medicine and Surgery, Medical School, University of Pisa, Via Roma 55, 56126 Pisa, Italy. ${ }^{2}$ CITHEFOR-EA 3452, Faculté de Pharmacie, Université de Lorraine, Nancy, France. ${ }^{3}$ Department of Surgery and Medical, Molecular, and Critical Area Pathology, Medical School, University of Pisa, Pisa, Italy. ${ }^{4}$ Life Science Institute, Scuola Superiore Sant'Anna, Pisa, Italy. ${ }^{5}$ Histopathology Department, University Hospital, Pisa, Italy.

\section{Acknowledgements}

The financial support by University of Pisa (Faculty of Medicine and Surgery Research Project 2012 and PRA Funds 2015) is kindly acknowledged.

\section{Competing interests}

The authors declare no conflict of interest.

Received: 10 August 2015 Accepted: 4 October 2015

Published online: 13 October 2015 


\section{References}

1. Ross R. Atherosclerosis is an inflammatory disease. Am Heart J. 1999;138(5 Pt 2):S419-20.

2. Shah PK. Mechanisms of plaque vulnerability and rupture. J Am Coll Cardiol. 2003;41(4 Suppl S):15S-22S.

3. Falk E. Pathogenesis of atherosclerosis. J Am Coll Cardiol. 2006;47(8 Suppl):C7-12

4. Johnson JL, Newby AC. Macrophage heterogeneity in atherosclerotic plaques. Curr Opin Lipidol. 2009;20(5):370-8.

5. Leitinger N, Schulman IG. Phenotypic polarization of macrophages in atherosclerosis. Arterioscler Thromb Vasc Biol. 2013;33(6):1120-6.

6. Zhou D, Huang C, Lin Z, Zhan S, Kong L, Fang C, Li J. Macrophage polarization and function with emphasis on the evolving roles of coordinated regulation of cellular signaling pathways. Cell Signal. 2014;26(2):192-7.

7. Stöger JL, Gijbels MJ, van der Velden S, Manca M, van der Loos CM, Biessen EA, et al. Distribution of macrophage polarization markers in human atherosclerosis. Atherosclerosis. 2012;225(2):461-8.

8. Chinetti-Gbaguidi G, Baron M, Bouhlel MA, Vanhoutte J, Copin C, Sebti Y, et al. Human atherosclerotic plaque alternative macrophages display low cholesterol handling but high phagocytosis because of distinct activities of the PPAR $\gamma$ and LXR $\alpha$ pathways. Circ Res. 2011;108(8):985-95.

9. Cho KY, Miyoshi H, Kuroda S, Yasuda H, Kamiyama K, Nakagawara J, et al. The phenotype of infiltrating macrophages influences arteriosclerotic plaque vulnerability in the carotid artery. J Stroke Cerebrovasc Dis. 2013;22(7):910-8.

10. Van Tits LJ, Stienstra R, van Lent PL, Netea MG, Joosten LA, Stalenhoef AF. Oxidized LDL enhances pro-inflammatory responses of alternatively activated M2 macrophages: a crucial role for Krüppel-like factor 2. Atherosclerosis. 2011;214(2):345-9.

11. Ruttmann E, Brant L, Concin H, Diem G, Rapp K, Ulmer H. Vorarlberg Health Monitoring and Promotion Program Study Group. Gammaglutamyltransferase as a risk factor for cardiovascular disease mortality: an epidemiological investigation in a cohort of 163,944 Austrian adults. Circulation. 2005;112(14):2130-7.

12. Emdin M, Passino C, Michelassi C, Titta F, L'abbate A, Donato L, et al. Prognostic value of serum gamma-glutamyl transferase activity after myocardial infarction. Eur Heart J. 2001;22(19):1802-7.

13. Franzini M, Bramanti E, Ottaviano V, Ghiri E, Scatena F, Barsacchi R, et al. A high performance gel filtration chromatography method for gammaglutamyltransferase fraction analysis. Anal Biochem. 2008;374(1):1-6.

14. Franzini M, Fornaciari I, Rong J, Larson MG, Passino C, Emdin M, et al. Correlates and reference limits of plasma gamma-glutamyltransferase fractions from the Framingham Heart Study. Clin Chim Acta. 2013:417:19-25.

15. Fornaciari I, Fierabracci V, Corti A, Aziz Elawadi H, Lorenzini E, Emdin M et al. Gamma-glutamyltransferase fractions in human plasma and bile: characteristic and biogenesis. PLoS One. 2014;9(2):e88532.

16. Franzini M, Corti A, Martinelli B, Del Corso A, Emdin M, Parenti GF, et al. Gamma-glutamyltransferase activity in human atherosclerotic plaquesbiochemical similarities with the circulating enzyme. Atherosclerosis. 2009;202(1):119-27

17. Wickham S, West MB, Cook PF, Hanigan MH. Gamma-glutamyl compounds: substrate specificity of gamma-glutamyl transpeptidase enzymes. Anal Biochem. 2011;414:208-14.

18. Angeli V, Tacito A, Paolicchi A, Barsacchi R, Franzini M, Baldassini R, et al. A kinetic study of gamma-glutamyltransferase (GGT)-mediated S-nitrosoglutathione catabolism. Arch Biochem Biophys. 2009;48:191-6.

19. Paolicchi A, Minotti G, Tonarelli P, Tongiani R, De Cesare D, Mezzetti A, et al. Gamma-glutamyl transpeptidase-dependent iron reduction and LDL oxidation-a potential mechanism in atherosclerosis. J Investig Med. 1999:47(3):151-60.

20. Dominici S, Paolicchi A, Lorenzini E, Maellaro E, Comporti M, Pieri L, et al. Gamma-glutamyltransferase-dependent prooxidant reactions: a factor in multiple processes. BioFactors. 2003;17(1-4):187-98.

21. Dominici S, Paolicchi A, Corti A, Maellaro E, Pompella A. Prooxidant reactions promoted by soluble and cell-bound gamma-glutamyltransferase activity. Methods Enzymol. 2005:401:484-501.

22. Pang JH, Jiang MJ, Chen YL, Wang FW, Wang DL, Chu SH, et al. Increased ferritin gene expression in atherosclerotic lesions. J Clin Invest. 1996:97(10):2204-12.

23. Hultberg B, Sjögren U. L-gamma-glutamyl transpeptidase activity in normal and leukemic leukocytes. Acta Haematol. 1980;63(3):132-5.
24. Khalaf MR, Hayhoe FG. Cytochemistry of gamma-glutamyltransferase in haemic cells and malignancies. Histochem J. 1987;19(6-7):385-95.

25. Emdin M, Passino C, Donato L, Paolicchi A, Pompella A. Serum gammaglutamyltransferase as a risk factor of ischemic stroke might be independent of alcohol consumption. Stroke. 2002;33(4):1163-4.

26. Paolicchi A, Emdin M, Ghliozeni E, Ciancia E, Passino C, Popoff G, et al. Images in cardiovascular medicine. Human atherosclerotic plaques contain gamma-glutamyl transpeptidase enzyme activity. Circulation. 2004:109(11):1440.

27. Pucci A, Franzini M, Matteucci M, Ceragioli S, Marconi M, Ferrari M, et al. b-Gamma-glutamyltransferase activity in human vulnerable carotid plaques. Atherosclerosis. 2014;237(1):307-13.

28. Wankowicz Z, Megyeri P, Issekutz A. Synergy between tumour necrosis factor alpha and interleukin-1 in the induction of polymorphonuclear leukocyte migration during inflammation. J Leukoc Biol. 1988;43(4):349-56.

29. Yoon JH, Kim KS, Kim HU, Linton JA, Lee JG. Effects of TNF-alpha and IL-1 beta on mucin, lysozyme, IL-6 and IL-8 in passage-2 normal human nasal epithelial cells. Acta Otolaryngol. 1999;119(8):905-10.

30. Tufvesson E, Westergren-Thorsson G. Alteration of proteoglycan synthesis in human lung fibroblasts induced by interleukin-1beta and tumor necrosis factor-alpha. J Cell Biochem. 2000;77(2):298-309.

31. Verreck FA, de Boer T, Langenberg DM, Hoeve MA, Kramer M, Vaisberg E, et al. Human IL-23-producing type 1 macrophages promote but IL10-producing type 2 macrophages subvert immunity to (myco)bacteria. Proc Natl Acad Sci USA. 2004;101(13):4560-5.

32. Verreck FA, de Boer T, Langenberg DM, van der Zanden L, OttenhoffTH. Phenotypic and functional profiling of human proinflammatory type-1 and anti-inflammatory type-2 macrophages in response to microbial antigens and IFN-gamma- and CD40L-mediated costimulation. J Leukoc Biol. 2006;79(2):285-93.

33. Lacey DC, Achuthan A, Fleetwood AJ, Dinh H, Roiniotis J, Scholz GM, et al. Defining GM-CSF- and macrophage-CSF-dependent macrophage responses by in vitro models. J Immunol. 2012;188(11):5752-65.

34. Matheeussen $V$, Waumans $Y$, Martinet W, Van Goethem S, Van der Veken $P$, Scharpé S, et al. Dipeptidyl peptidases in atherosclerosis: expression and role in macrophage differentiation, activation and apoptosis. Basic Res Cardiol. 2013;108(3):350.

35. Barnett HJ, Taylor DW, Eliasziw M, Fox AJ, Ferguson GG, Haynes RB, et al. Benefit of carotid endarterectomy in patients with symptomatic moderate or severe stenosis. North American Symptomatic Carotid Endarterectomy Trial Collaborators. N Engl J Med. 1998;339(20):1415-25.

36. Hanigan MH, Frierson HF Jr. Immunohistochemical detection of gammaglutamyl transpeptidase in normal human tissue. J Histochem Cytochem. 1996;44(10):1101-8

37. Corti A, Franzini M, Cianchetti S, Bergamini G, Lorenzini E, Melotti P, et al. Contribution by polymorphonucleate granulocytes to elevated gammaglutamyltransferase in cystic fibrosis sputum. PLoS One. 2012;7(4):e34772.

38. Franzini M, Ottaviano V, Fierabracci V, Bramanti E, Zyw L, Barsacchi R, et al. Fractions of plasma gamma-glutamyltransferase in healthy individuals: reference values. Clin Chim Acta. 2008:395(1-2):188-9.

39. Huseby NE, Strömme JH. Practical points regarding routine determination of gamma-glutamyl transferase (gamma-GT) in serum with a kinetic method at 37 degrees C. Scand J Clin Lab Invest. 1974;34(4):357-63.

40. Niida S, Kawahara M, Ishizuka Y, Ikeda Y, Kondo T, Hibi T, et al. Gamma-glutamyltranspeptidase stimulates receptor activator of nuclear factor-kappaB ligand expression independent of its enzymatic activity and serves as a pathological bone-resorbing factor. J Biol Chem. 2004;279(7):5752-6.

41. Evjen $\mathrm{G}$, Huseby NE. Characterization of the carbohydrate moiety of human gamma-glutamyltransferases using lectin-blotting and glycosidase treatment. Clin Chim Acta. 1992;209(1-2):27-34.

42. Pshezhetsky AV, Ashmarina M. Lysosomal multienzyme complex: biochemistry, genetics, and molecular pathophysiology. Prog Nucleic Acid Res Mol Biol. 2001;69:81-114.

43. Miller AM, Sandler E, Kobb SM, Eastgate J, Zucali J. Hematopoietic growth factor induction of gamma-glutamyl transferase in the KG-1 myeloid cell line. Exp Hematol. 1993;21(1):9-15.

44. Guidez F, Li AC, Horvai A, Welch JS, Glass CK. Differential utilization of Ras signaling pathways by macrophage colony-stimulating factor (CSF) and granulocyte-macrophage CSF receptors during macrophage differentiation. Mol Cell Biol. 1998:18(7):3851-61. 
45. Ebner K, Bandion A, Binder BR, de Martin R, Schmid JA. GMCSF activates NF-kappaB via direct interaction of the GMCSF receptor with IkappaB kinase beta. Blood. 2003;102(1):192-9.

46. Rego SL, Helms RS, Dréau D. Breast tumor cell TACE-shed MCSF promotes pro-angiogenic macrophages through NF-кB signaling. Angiogenesis. 2014;17(3):573-85.

47. Fleetwood AJ, Dinh H, Cook AD, Hertzog PJ, Hamilton JA. GM-CSF- and M-CSF-dependent macrophage phenotypes display differential dependence on type I interferon signaling. J Leukoc Biol. 2009;86(2):411-21.

48. Pandur S, Pankiv S, Johannessen M, Moens U, Huseby NE. Gammaglutamyltransferase is upregulated after oxidative stress through the Ras signal transduction pathway in rat colon carcinoma cells. Free Radic Res. 2007;41(12):1376-84.

49. Daubeuf S, Accaoui MJ, Pettersen I, Huseby NE, Visvikis A, Galteau MM Differential regulation of gamma-glutamyltransferase mRNAs in four human tumour cell lines. Biochim Biophys Acta. 2001;1568(1):67-73.

50. Reuter S, Schnekenburger M, Cristofanon S, Buck I, Teiten MH, Daubeuf $\mathrm{S}$, et al. Tumor necrosis factor alpha induces gamma-glutamyltransferase expression via nuclear factor-kappaB in cooperation with Sp1. Biochem Pharmacol. 2009;77(3):397-411.

51. Driessler F, Venstrom K, Sabat R, Asadullah K, Schottelius AJ. Molecular mechanisms of interleukin-10-mediated inhibition of NF-kappaB activity: a role for p50. Clin Exp Immunol. 2004;135(1):64-73.

52. Dhingra S, Sharma AK, Arora RC, Slezak J, Singal PK. IL-10 attenuates TNF-alpha-induced NF kappaB pathway activation and cardiomyocyte apoptosis. Cardiovasc Res. 2009;82(1):59-66.

53. Geissmann F, Manz MG, Jung S, Sieweke MH, Merad M, Ley K. Development of monocytes, macrophages, and dendritic cells. Science. 2010;327(5966):656-61.

54. Martinez FO, Sica A, Mantovani A, Locati M. Macrophage activation and polarization. Front Biosci. 2008;13:453-61.

55. Heisterkamp N, Groffen J, Warburton D, Sneddon TP. The human gammaglutamyltransferase gene family. Hum Genet. 2008;123(4):321-32.

\section{Submit your next manuscript to BioMed Central} and take full advantage of:

- Convenient online submission

- Thorough peer review

- No space constraints or color figure charges

- Immediate publication on acceptance

- Inclusion in PubMed, CAS, Scopus and Google Scholar

- Research which is freely available for redistribution

Submit your manuscript at

www.biomedcentral.com/submit

C Biomed Central 